\title{
The Minimum Spectral Radius of an Edge-Removed Network: A Hypercube Perspective
}

\author{
Yingbo Wu, ${ }^{1}$ Tianrui Zhang, ${ }^{1}$ Shan Chen, ${ }^{2}$ and Tianhui Wang ${ }^{1}$ \\ ${ }^{1}$ School of Software Engineering, Chongqing University, Chongqing 400044, China \\ ${ }^{2}$ School of Mechanical Engineering, Chongqing University, Chongqing 400044, China \\ Correspondence should be addressed to Yingbo Wu; wyb@cqu.edu.cn
}

Received 15 December 2016; Revised 17 February 2017; Accepted 28 March 2017; Published 19 April 2017

Academic Editor: Yong Deng

Copyright (c) 2017 Yingbo Wu et al. This is an open access article distributed under the Creative Commons Attribution License, which permits unrestricted use, distribution, and reproduction in any medium, provided the original work is properly cited.

\begin{abstract}
The spectral radius minimization problem (SRMP), which aims to minimize the spectral radius of a network by deleting a given number of edges, turns out to be crucial to containing the prevalence of an undesirable object on the network. As the SRMP is NP-hard, it is very unlikely that there is a polynomial-time algorithm for it. As a result, it is proper to focus on the development of effective and efficient heuristic algorithms for the SRMP. For that purpose, it is appropriate to gain insight into the pattern of an optimal solution to the SRMP by means of checking some regular networks. Hypercubes are a celebrated class of regular networks. This paper empirically studies the SRMP for hypercubes with two/three/four missing edges. First, for each of the three subproblems of the SRMP, a candidate for the optimal solution is presented. Second, it is shown that the candidate is optimal for small-sized hypercubes, and it is shown that the proposed candidate is likely to be optimal for medium-sized hypercubes. The edges in each candidate are evenly distributed over the network, which may be a common feature of all symmetric networks and hence is instructive in designing effective heuristic algorithms for the SRMP.
\end{abstract}

\section{Introduction}

The epidemic modeling is recognized as an effective approach to the understanding of propagation process of objects over a network [1,2]. For instance, epidemic models help us understand the key factors that affect the prevalence of malware [3-8]. The speed and extent of spread of an epidemic on a network depend largely on the structure of the network; whether the epidemic goes viral depends on whether the spectral radius of the network exceeds a threshold [9-14]. Therefore, reducing the spectral radius of a network by removing a set of edges is an effective approach to the containment of the prevalence of an undesirable epidemic on the network. The spectral radius minimization problem (SRMP) aims to remove a given number of edges of a network so that the spectral radius of the resulting network attains the minimum. As the SRMP is NP-hard [15], it is much unlikely that there be a polynomial-time algorithm for it. As thus, a number of heuristic algorithms for the SRMP have been proposed [15-19]. In most situations, these heuristics are ineffective, because they produce nonoptimal solutions rather than optimal solutions. For the purpose of developing effective heuristic algorithms for the SRMP, it is appropriate to gain insight into the pattern of an optimal solution to the SRMP by means of checking some regular networks. Recently, Yang et al. [20] studied the SRMP for 2D tori.

Hypercubes are a class of regular networks [21]. Due to remarkable advantages in communication [22-25], fault tolerant communication [26-30], fault diagnosis [31-34], and parallel computation $[35,36]$, hypercubes have been widely adopted as the underlying interconnection network in multicomputer systems [37]. To our knowledge, the SRMP for hypercubes is still unsolved.

This paper addresses three subproblems of the SRMP, where two/three/four edges are removed from a hypercube, respectively. First, for each of the three subproblems of the SRMP, a candidate optimal solution is presented. Second, it is shown that the candidate is optimal for small-sized hypercubes, and it is shown that the proposed candidate is likely to be optimal for medium-sized hypercubes. The edges 


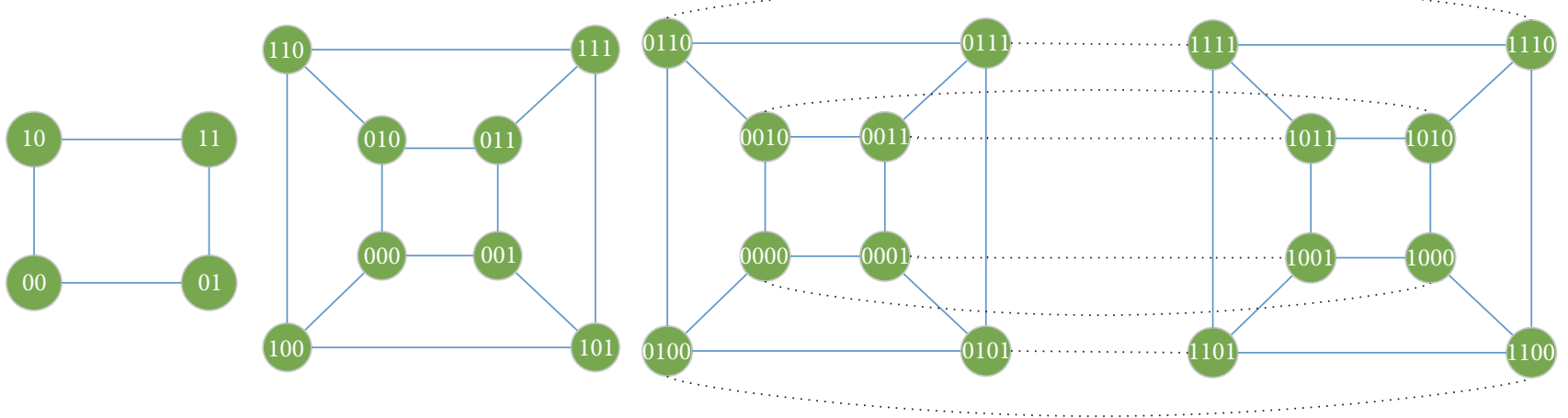

FIGURE 1: Three examples of $H_{n}$.
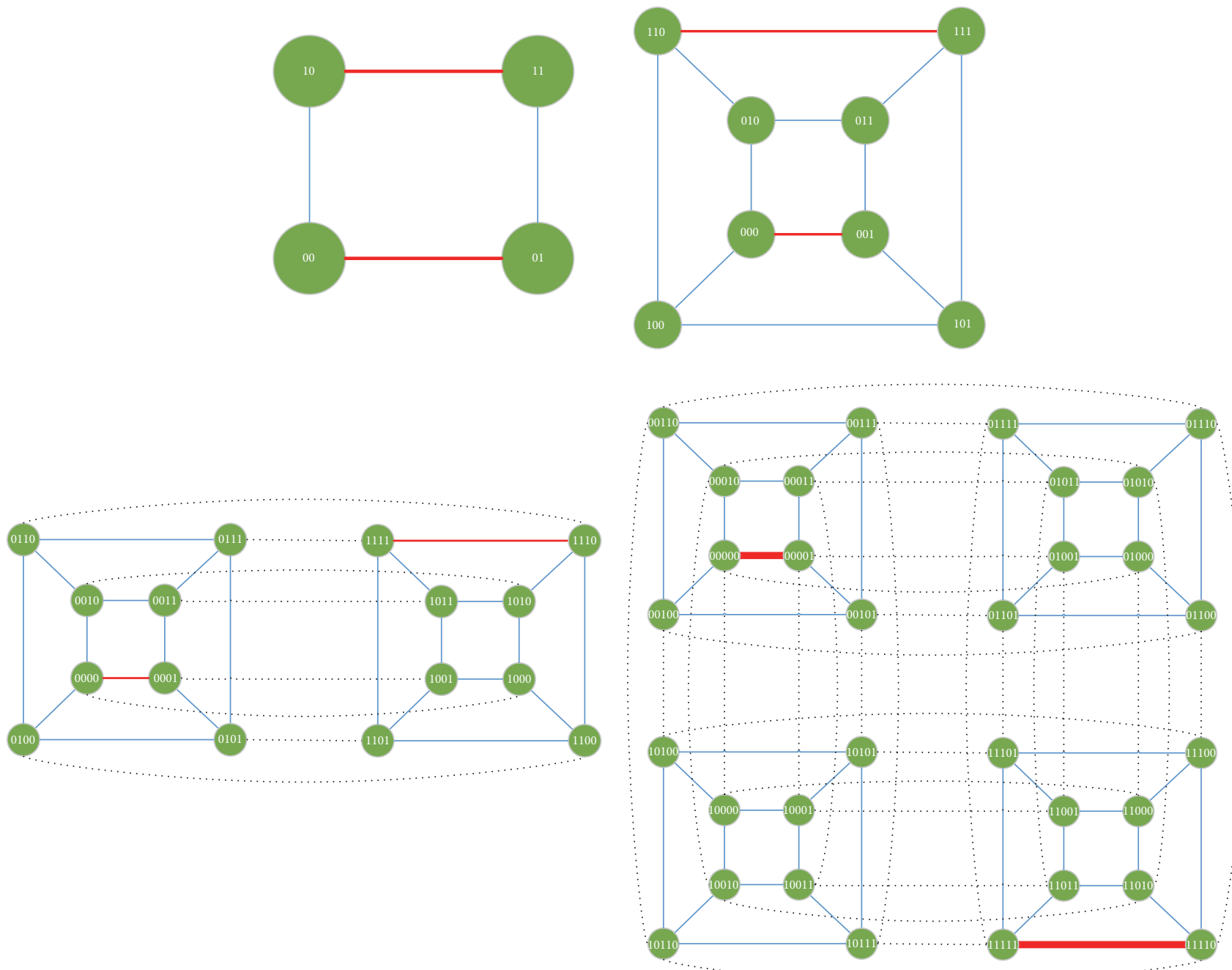

FIgURE 2: The proposed candidate in $H_{n}$.

in each candidate are evenly distributed over the network, which may be a common feature of all symmetric networks and hence is instructive in designing effective heuristic algorithms for the SRMP.
The remaining materials are organized in this fashion: the preliminary knowledge is given in Section 2. Section 3 presents the main results of this work. Finally, Section 4 summarizes this work. 


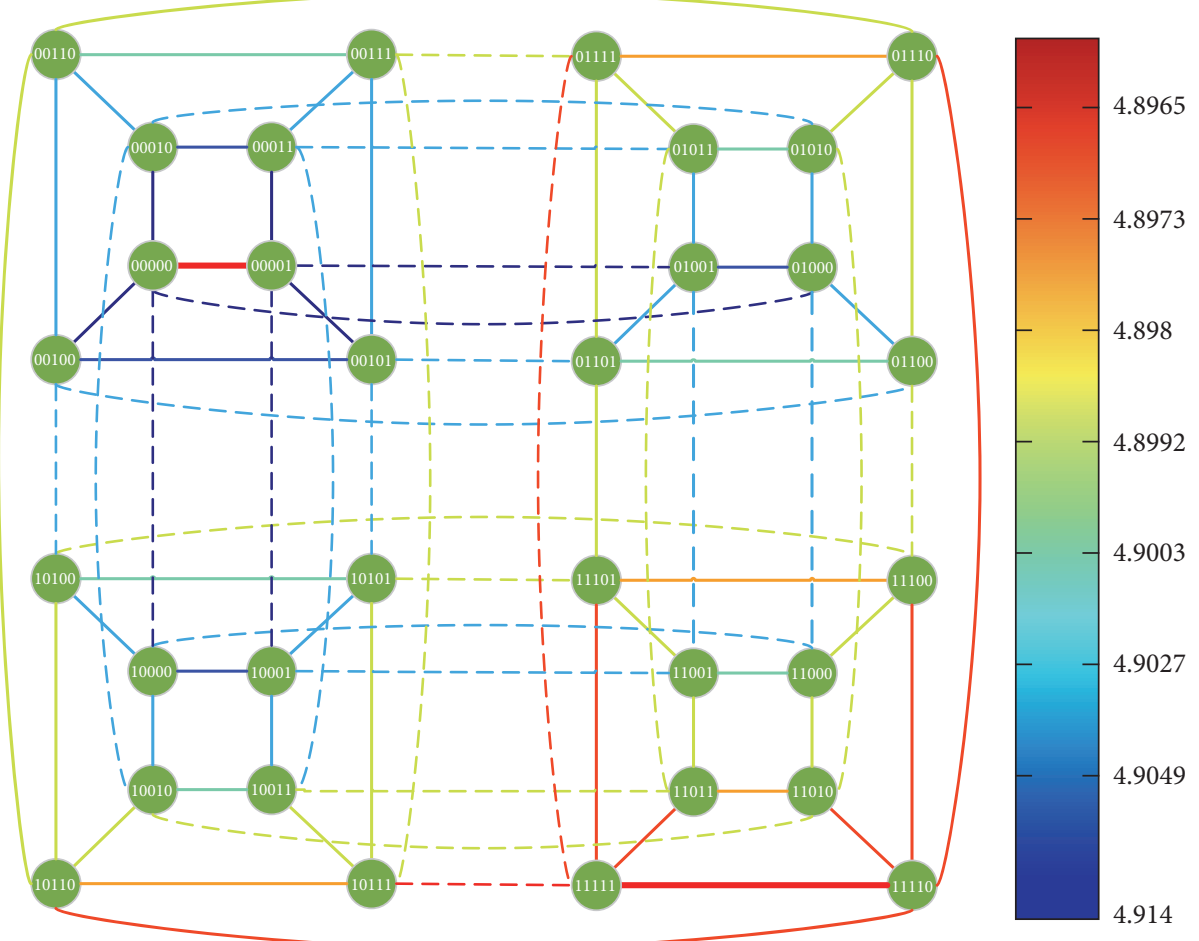

FIGURE 3: Assume that the red edge in the upper-left 3D subcube of $H_{5}$ is the first deleted edge and each of the remaining edges is a candidate for the second deleted edge. The spectral radius of the surviving network formed by deleting each of the candidate edges from $H_{5}$ is shown.
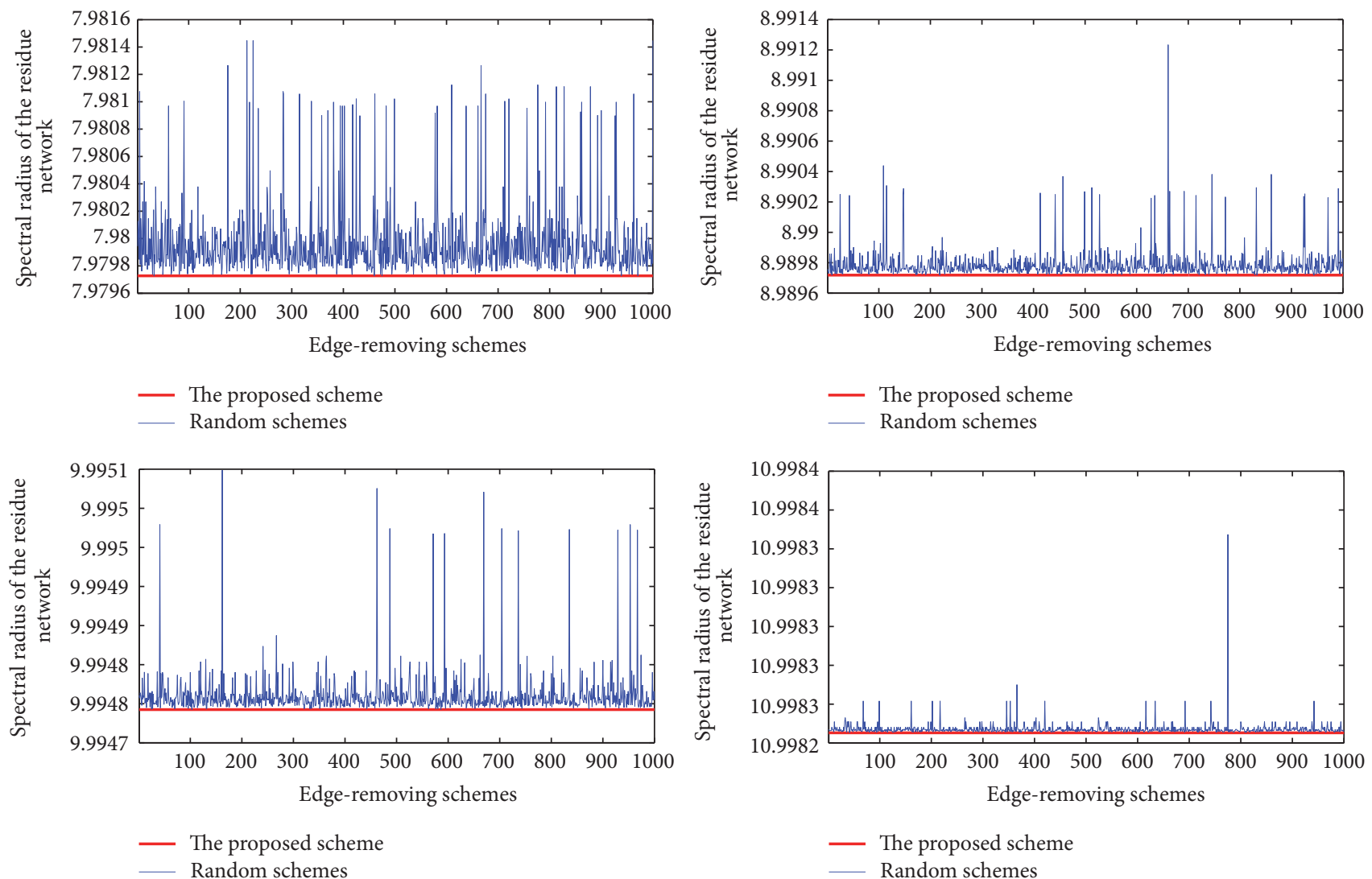

- The proposed scheme

_ Random schemes

FIgURE 4: The proposed candidate (red) versus $10^{3}$ random candidates (blue). 

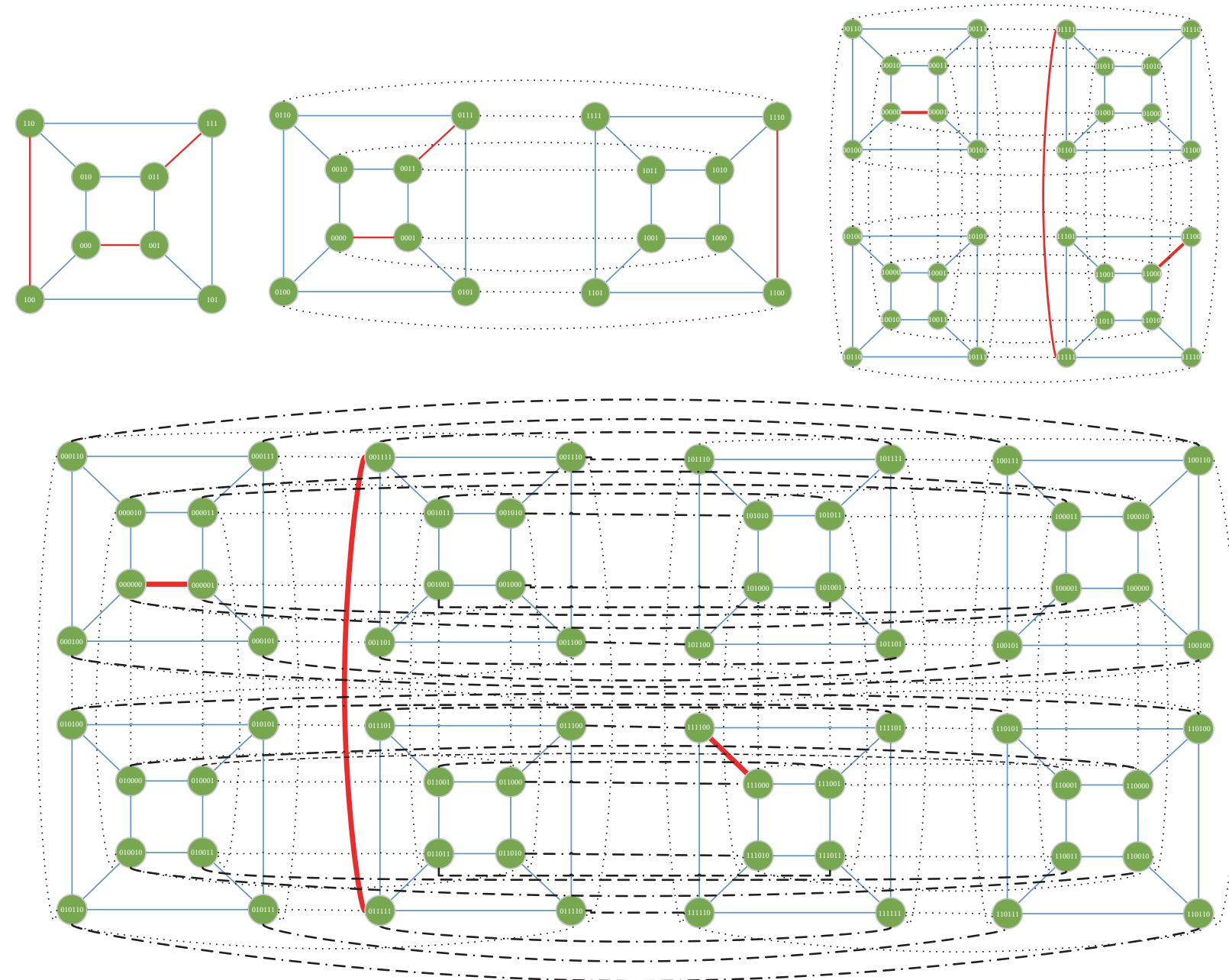

FIGURE 5: The proposed candidate in $H_{n}$.

\section{Preliminaries}

For fundamental knowledge on the spectral radius of a network, see $[38,39]$. The SRMP is formulated as follows: given a network $G=(V, E)$ and a positive integer $k$, find a set of $k$ edges of $G$ so that the surviving network obtained by removing the set of edges from the network achieves the minimum spectral radius.

An $n$-dimensional cube ( $n$-D cube, for short), denoted by $H_{n}$, is a network $G=(V, E)$, where there is a one-to-one correspondance $\phi$ from $V$ to the set of all 0-1 binary strings of length $n$ so that node $u$ is adjacent to node $v$ if and only if $\phi(u)$ differs from $\phi(v)$ in exactly one bit position. In what follows, it is always assumed that the nodes of a hypercube have been labelled with $0-1$ strings in this way. See Figure 1 for three small-sized hypercubes.

An $n$-D cube can also be defined in a recursive way as follows. (1) A 0-D cube is a graph on a single node. (2) For $n \geq 1$, an $n$-D cube is built from two copies of an $(n-1)$-D cube in this way: connect each node in one copy to the same node in the other copy.

\section{Main Results}

This section considers the optimal scheme of deleting two/three/four edges from $H_{n}$, respectively.

3.1. Deleting Two Edges. Firstly, we consider a subproblem of the SRMP, denoted by SRMP-H2, for which two edges will be deleted from a hypercube. Let us present a candidate for the optimal solution to the SRMP-H2 as follows, where $n$ denotes the dimension of the hypercube:

$$
\begin{aligned}
& e_{1}=\left\{0^{n}, 0^{n-1} 1\right\}, \\
& e_{2}=\left\{1^{n}, 1^{n-1} 0\right\} .
\end{aligned}
$$

Figure 2 shows the proposed candidate in $\mathrm{H}_{2}, \mathrm{H}_{3}, \mathrm{H}_{4}$, and $\mathrm{H}_{5}$, respectively.

For $2 \leq n \leq 7$, it follows by exhaustive search that the proposed candidate is optimal. For instance, assume that the red edge in the upper-left $3 \mathrm{D}$ subcube of $H_{5}$ is the first deleted edge, and each of the remaining edges is a candidate for 

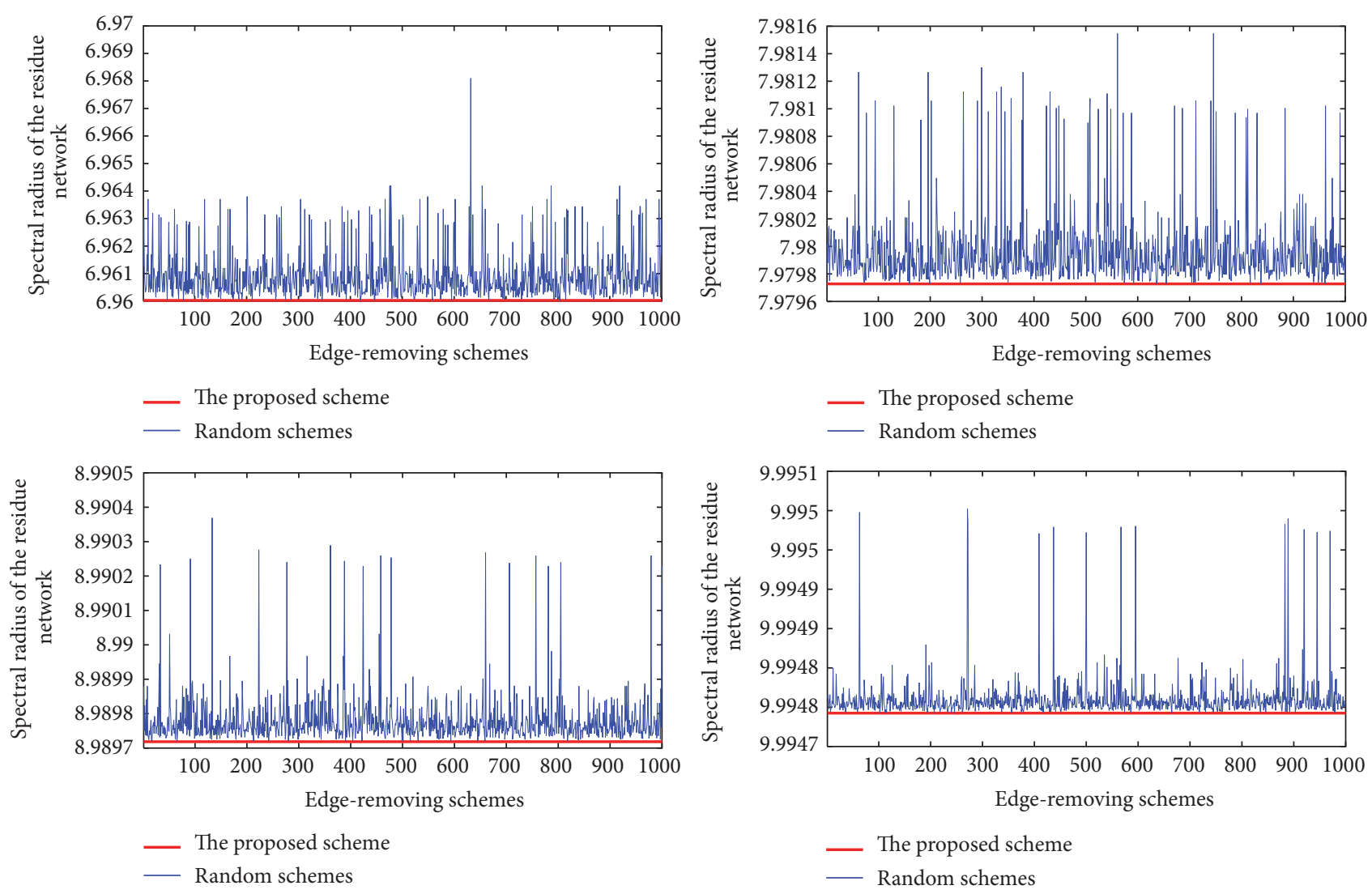

FIgURE 6: The proposed candidate (red) versus $10^{3}$ random candidates (blue).

the second deleted edge. The spectral radius of the surviving network formed by deleting each of the candidate edges from $\mathrm{H}_{5}$ is shown in Figure 3. It can be seen that the larger the distance between the two edges, the smaller the spectral radius of the surviving network. At the extreme, the proposed candidate is optimal.

For $8 \leq n \leq 11$, the proposed candidate is compared with $10^{3}$ random candidates in terms of the spectral radius of the surviving network; see Figure 4. It is concluded that the proposed candidate is optimal among these candidates.

Therefore, we propose the following conjecture.

Conjecture 1. For all $n \geq 2$, the proposed candidate is an optimal solution to the SRMP-H2.

3.2. Deleting Three Edges. Secondly, we consider a subproblem of the SRMP problem, denoted by SRMP-H3, for which three edges will be removed from a hypercube. Let us present a candidate for the optimal solution to the SRMP-H3 as follows, where $n$ denotes the dimension of the hypercube, $c=\lfloor(n+1) / 3\rfloor$ :

$$
\begin{aligned}
& e_{1}=\left\{0^{n}, 0^{n-1} 1\right\}, \\
& e_{2}=\left\{0^{n-2 c} 1^{2 c}, 0^{n-2 c-1} 1^{2 c+1}\right\}, \\
& e_{3}=\left\{1^{n-c-1} 0^{c+1}, 1^{n-c} 0^{c}\right\} .
\end{aligned}
$$

Figure 5 shows the proposed candidate in $H_{3}, H_{4}, H_{5}$, and $H_{6}$, respectively.

For $2 \leq n \leq 6$, it follows by exhaustive search that the proposed candidate is optimal. For $7 \leq n \leq 10$, the proposed candidate is compared with $10^{3}$ random candidates; see Figure 6. It is concluded that the proposed candidate is optimal among these candidates. Therefore, we propose the following conjecture.

Conjecture 2. For all $n \geq 3$, the proposed candidate is an optimal solution to the SRMP-H3.

3.3. Deleting Four Edges. Finally, consider a subproblem of the SRMP, denoted by SRMP-H4, for which four edges will be deleted from a hypercube. Let us present a candidate to the optimal solution to the SRMP-H4 as follows, where $n$ denotes the dimension of the hypercube, $c=\lfloor(n-1) / 3\rfloor$ :

If $n \equiv 0,2 \bmod 3$, then

$$
\begin{aligned}
& e_{1}=\left\{0^{n}, 0^{n-1} 1\right\}, \\
& e_{2}=\left\{0^{n-2 c-2} 1^{2 c+2}, 0^{n-2 c-2} 1^{2 c+1} 0\right\}, \\
& e_{3}=\left\{1^{n-2 c-2} 0^{c} 1^{c+1} 0,1^{n-2 c-2} 0^{c} 1^{c} 0^{2}\right\}, \\
& e_{4}=\left\{1^{n-c-2} 0^{c+1} 1,1^{n-c-2} 0^{c} 1^{2}\right\} .
\end{aligned}
$$



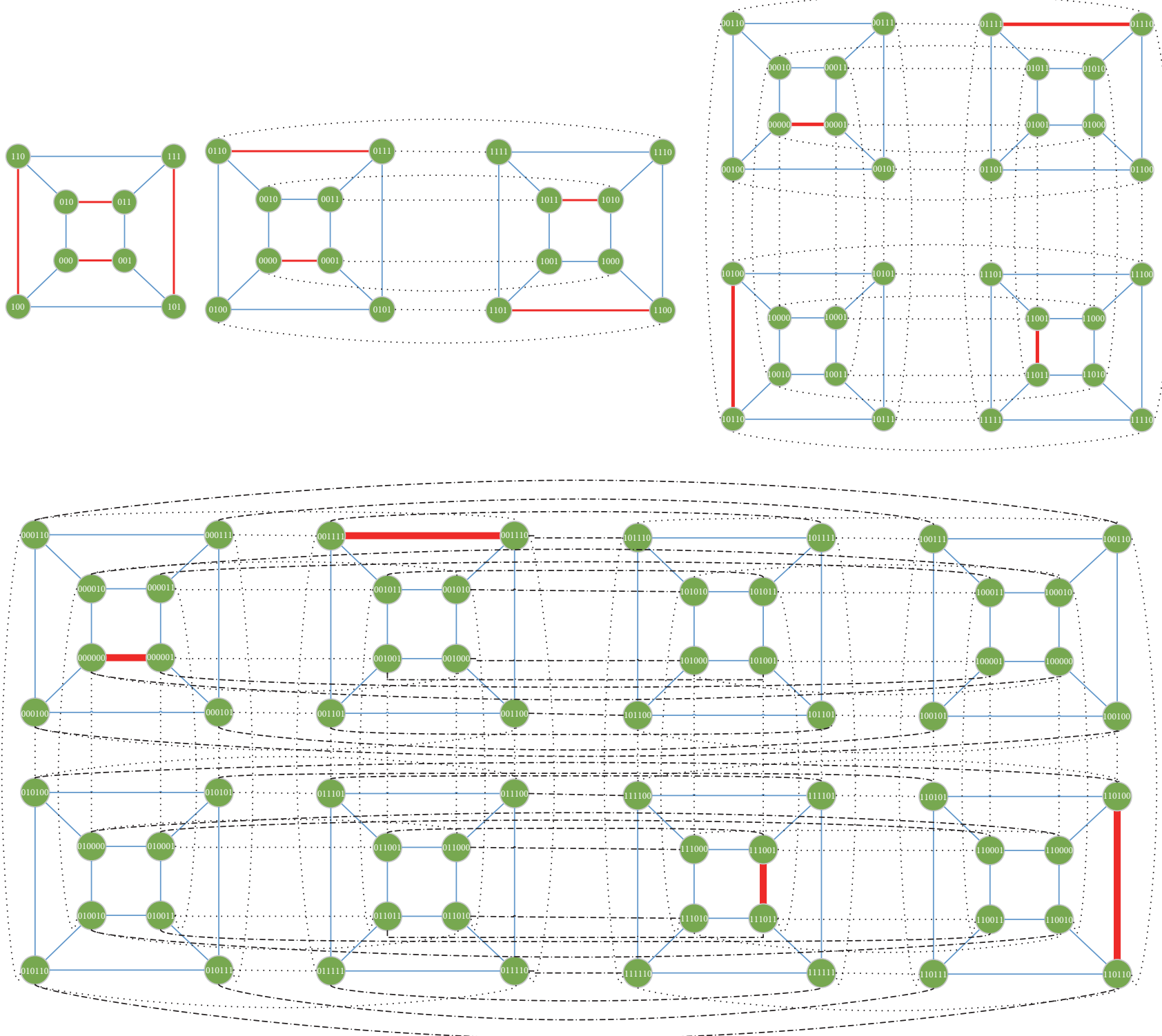

FIGURE 7: The proposed candidate in $H_{n}$.

If $n \equiv 1 \bmod 3$, then

$$
\begin{aligned}
& e_{1}=\left\{0^{n}, 0^{n-1} 1\right\}, \\
& e_{2}=\left\{0^{n-2 c-1} 1^{2 c+1}, 0^{n-2 c-1} 1^{2 c} 0\right\}, \\
& e_{3}=\left\{1^{n-2 c-1} 0^{c} 1^{c+1}, 1^{n-2 c-1} 0^{c} 1^{c} 0\right\}, \\
& e_{4}=\left\{1^{n-c-1} 0^{c+1}, 1^{n-c-1} 0^{c} 1\right\} .
\end{aligned}
$$

Figure 7 shows the proposed candidate in $\mathrm{H}_{3}, \mathrm{H}_{4}, \mathrm{H}_{5}$, and $H_{6}$, respectively.

For $3 \leq n \leq 6$, it follows by exhaustive search that the proposed candidate is an optimal solution to the SRMP-H4 problem. For $7 \leq n \leq 10$, the proposed candidate is compared with $10^{3}$ random candidates; see Figure 8 . It is concluded that the proposed candidate is optimal among these candidates. Therefore, we propose the following conjecture.

Conjecture 3. For all $n \geq 3$, the proposed candidate is an optimal solution to the SRMP-H4.

\section{Summary}

This paper has addressed the spectral radius minimization problem for hypercubes. Given the number of edges to be deleted, a candidate for the optimal solution has been presented. For small-sized hypercubes, the proposed candidate has been shown to be optimal. For medium-sized hypercubes, it has been shown that the proposed candidate is likely to be optimal. Due to the symmetry of hypercubes, there are multiple optimal solutions for each of the subproblems. The 

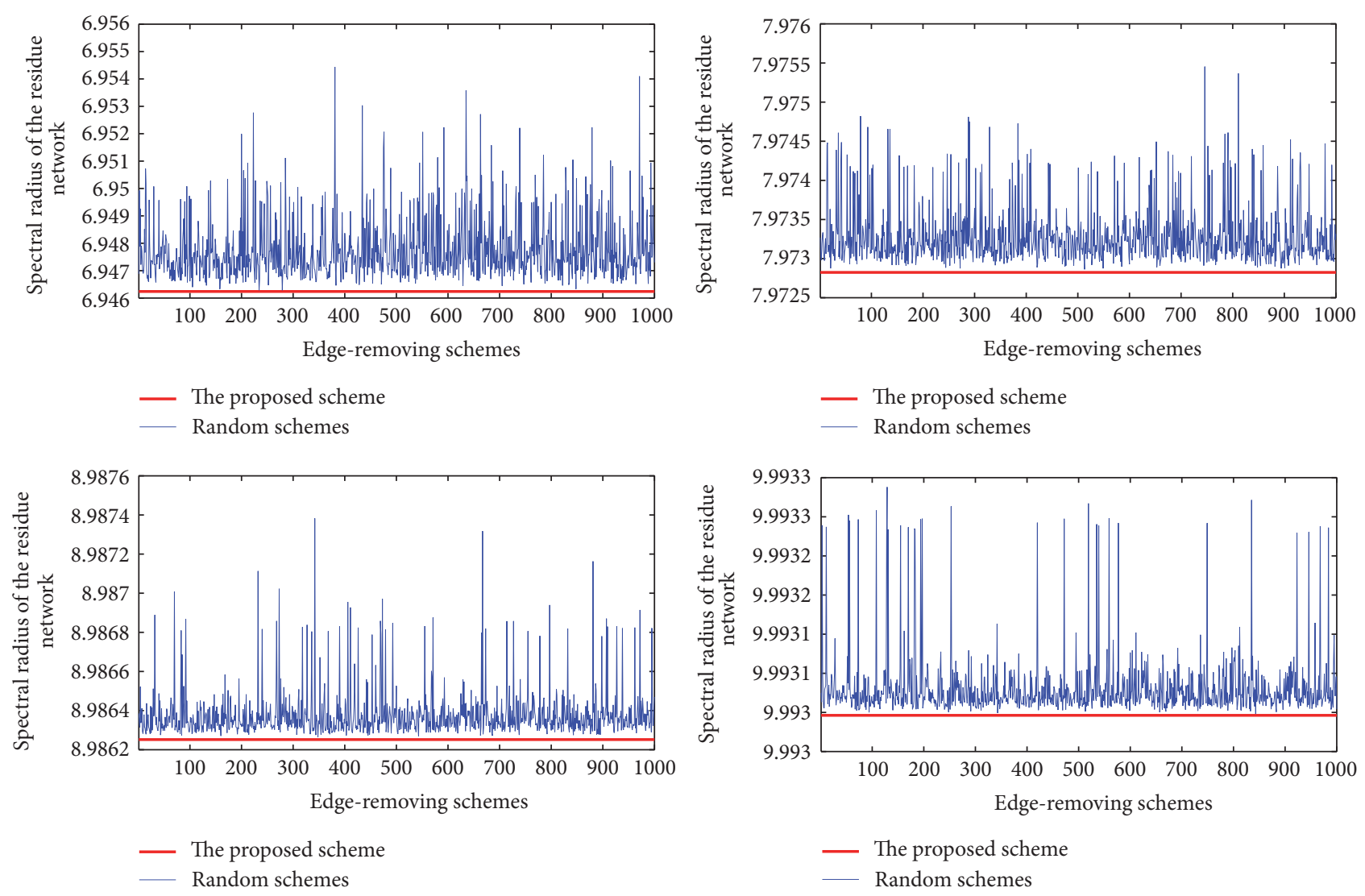

FIgURE 8: The proposed candidate (red) versus $10^{3}$ random candidates (blue).

experimental results show that, up to isomorphism, all of the optimal solutions are identical. By observing the pattern of the proposed candidate, it has been speculated that, for any symmetric network, the edges in an optimal solution are always evenly distributed.

Towards this direction, some researches are yet to be done. First, the proposed conjectures need a proof. Second, this work should be extended to asymmetric networks such as the hypercube-like networks [40,41], the small-world networks [42], the scale-free networks [43, 44], and the general networks $[45,46]$. Last, the effectiveness of heuristics for the spectral radius minimization problem must be improved.

\section{Conflicts of Interest}

The authors declare that there are no conflicts of interest regarding the publication of this paper.

\section{Acknowledgments}

This work was supported by Science and Technology Support Program of China (Grant no. 2015BAF05B03), Natural Science Foundation of China (Grants nos. 61572006, 71301177), Basic and Advanced Research Program of Chongqing (Grant no. cstc2013jcyjA1658), and Fundamental Research Funds for the Central Universities (Grant no. 106112014CDJZR008823).

\section{References}

[1] Z. Ma, Y. Zhou, and J. Wu, Modeling and Dynamics of Infectious Diseases, Higher Education Press, Beijing, China, 2009.

[2] M. Draief and L. Massoulié, Epidemics and Rumours in Complex Networks, Cambridge University Press, 2010.

[3] J. O. Kephart and S. R. White, "Directed-graph epidemiological models of computer viruses," in Proceedings of the IEEE Computer Society Symposium on Research of Security and Privacy, pp. 343-359, 1991.

[4] J. O. Kephart, S. R. White, and D. M. Chess, "Computers and epidemiology," IEEE Spectrum, vol. 30, no. 5, pp. 20-26, 1993.

[5] C. Gao, J. Liu, and N. Zhong, "Network immunization with distributed autonomy-oriented entities," IEEE Transactions on Parallel and Distributed Systems, pp. 1222-1229, 2011.

[6] L.-X. Yang, X. Yang, J. Liu, Q. Zhu, and C. Gan, "Epidemics of computer viruses: a complex-network approach," Applied Mathematics and Computation, vol. 219, no. 16, pp. 8705-8717, 2013.

[7] C. Gao and J. Liu, "Modeling and restraining mobile virus propagation," IEEE Transactions on Mobile Computing, vol. 12, no. 3, pp. 529-541, 2013.

[8] L.-X. Yang and X. Yang, "A novel virus-patch dynamic model," PLoS ONE, vol. 10, no. 9, Article ID e0137858, 2015.

[9] M. Draief, A. Ganesh, and L. Massoulié, "Thresholds for virus spread on networks," The Annals of Applied Probability, vol. 18, no. 2, pp. 359-378, 2008. 
[10] P. Van Mieghem, J. Omic, and R. Kooij, "Virus spread in networks," IEEE/ACM Transactions on Networking, vol. 17, no. 1, pp. 1-14, 2009.

[11] P. Van Mieghem, "The $N$-intertwined SIS epidemic network model," Computing, vol. 93, no. 2-4, pp. 147-169, 2011.

[12] F. D. Sahneh, F. N. Chowdhury, and C. M. Scoglio, "On the existence of a threshold for preventive behavioral responses to suppress epidemic spreading," Scientific Reports, vol. 2, article 00632, 2012.

[13] F. D. Sahneh, C. Scoglio, and P. Van Mieghem, "Generalized epidemic mean-field model for spreading processes over multilayer complex networks," IEEE/ACM Transactions on Networking, vol. 21, no. 5, pp. 1609-1620, 2013.

[14] L. X. Yang and X. Yang, "The effect of network topology on the spread of computer viruses: a modelling study," International Journal of Computer Mathematics, 2016.

[15] P. Van Mieghem, D. Stevanović, F. Kuipers et al., "Decreasing the spectral radius of a graph by link removals," Physical Review E-Statistical, Nonlinear, and Soft Matter Physics, vol. 84, no. 1, Article ID 016101, 2011.

[16] J. G. Restrepo, E. Ott, and B. R. Hunt, "Approximating the largest eigenvalue of network adjacency matrices," Physical Review E. Statistical, Nonlinear, and Soft Matter Physics, vol. 76, no. 5, 056119, 6 pages, 2007.

[17] A. Milanese, J. Sun, and T. Nishikawa, "Approximating spectral impact of structural perturbations in large networks," Physical Review E - Statistical, Nonlinear, and Soft Matter Physics, vol. 81, no. 4, Article ID 046112, 2010.

[18] P. Van Mieghem, H. Wang, X. Ge, S. Tang, and F. A. Kuipers, "Influence of assortativity and degree-preserving rewiring on the spectra of networks," European Physical Journal B, vol. 76, no. 4, pp. 643-652, 2010.

[19] C. Li, H. Wang, and P. Van Mieghem, "Degree and principal eigenvectors in complex networks," Lecture Notes in Computer Science (including subseries Lecture Notes in Artificial Intelligence and Lecture Notes in Bioinformatics), vol. 7289, no. 1, pp. 149160, 2012.

[20] X. Yang, P. Li, L.-X. Yang, and Y. Wu, "Reducing the spectral radius of a torus network by link removal," PLoS ONE, vol. 11, no. 5, Article ID e0155580, 2016.

[21] J. Xu, A First Course in Graph Theory, Science Press, 2015.

[22] Y. Saad and M. H. Schultz, "Data communication in hypercubes," Journal of Parallel and Distributed Computing, vol. 6, no. 1, pp. 115-135, 1989.

[23] T. Bier and K.-F. Loe, "Embedding of binary trees into hypercubes," Journal of Parallel and Distributed Computing, vol. 6, no. 3, pp. 679-691, 1989.

[24] V. Heun and E. W. Mayr, "Efficient dynamic embeddings of binary trees into hypercubes," Journal of Algorithms. Cognition, Informatics and Logic, vol. 43, no. 1, pp. 51-84, 2002.

[25] C.-N. Lai, "Optimal construction of all shortest node-disjoint paths in hypercubes with applications," IEEE Transactions on Parallel and Distributed Systems, vol. 23, no. 6, pp. 1129-1134, 2012.

[26] P. J. Yang, S. B. Tien, and C. S. Raghavendra, "Reconfiguration of rings and meshes in faulty hypercubes," Journal of Parallel and Distributed Computing, vol. 22, no. 1, pp. 96-106, 1994.

[27] D. Xiang, Y. Zhang, and J.-G. Sun, "Unicast-based faulttolerant multicasting in wormhole-routed hypercubes," Journal of Systems Architecture, vol. 54, no. 12, pp. 1164-1178, 2008.
[28] W. Yang and J. Meng, "Generalized measures of fault tolerance in hypercube networks," Applied Mathematics Letters, vol. 25, no. 10, pp. 1335-1339, 2012.

[29] T.-L. Kung, C.-K. Lin, and L.-H. Hsu, "On the maximum number of fault-free mutually independent Hamiltonian cycles in the faulty hypercube," Journal of Combinatorial Optimization, vol. 27, no. 2, pp. 328-344, 2014.

[30] J.-J. Liu and Y.-L. Wang, "Hamiltonian cycles in hypercubes with faulty edges," Information Sciences. An International Journal, vol. 256, pp. 225-233, 2014.

[31] S.-L. Peng, C.-K. Lin, J. J. Tan, and L.-H. Hsu, “The g-goodneighbor conditional diagnosability of hypercube under PMC model," Applied Mathematics and Computation, vol. 218, no. 21, pp. 10406-10412, 2012.

[32] M.-C. Yang, "Conditional diagnosability of matching composition networks under the $\mathrm{MM}^{*}$ model," Information Sciences, vol. 233, pp. 230-243, 2013.

[33] C.-K. Lin, T.-L. Kung, and J. J. Tan, "An algorithmic approach to conditional-fault local diagnosis of regular multiprocessor interconnected systems under the PMC model," IEEE Transactions on Computers, vol. 62, no. 3, pp. 439-451, 2013.

[34] P.-L. Lai, "Adaptive system-level diagnosis for hypercube multiprocessors using a comparison model," Information Sciences, vol. 252, pp. 118-131, 2013.

[35] K. W. Ryu and J. Jájá, "Efficient algorithms for list ranking and for solving graph problems on the hypercube," IEEE Transactions on Parallel and Distributed Systems, vol. 1, no. 1, pp. 83-90, 1990.

[36] O. H. Ibarra and M. H. Kim, "Fast parallel algorithms for solving triangular systems of linear equations on the hypercube," Journal of Parallel and Distributed Computing, vol. 20, no. 3, pp. 303-316, 1994.

[37] A. Grama, A. Gupta, G. Karypis, and V. Kumar, Introduction to Parallel Computing, The Benjamin/Cummings Publishing Company, Inc., 2nd edition, 1994.

[38] P. Van Mieghem, Graph Spectra for Complex Networks, Cambridge University Press, 2012.

[39] D. Stevanovic, Spectral Radius of Graphs, Elsevier, 2015.

[40] J. Fan, X. Jia, X. Liu, S. Zhang, and J. Yu, "Efficient unicast in bijective connection networks with the restricted faulty node set," Information Sciences, vol. 181, no. 11, pp. 2303-2315, 2011.

[41] J. Fan, X. Jia, B. Cheng, and J. Yu, "An efficient faulttolerant routing algorithm in bijective connection networks with restricted faulty edges," Theoretical Computer Science, vol. 412, no. 29, pp. 3440-3450, 2011.

[42] D. J. Watts and S. H. Strogatz, "Collective dynamics of 'smallworld' networks," Nature, vol. 393, no. 6684, pp. 440-442, 1998.

[43] A.-L. Barabási and R. Albert, "Emergence of scaling in random networks," American Association for the Advancement of Science. Science, vol. 286, no. 5439, pp. 509-512, 1999.

[44] R. Albert and A.-L. Barabási, "Statistical mechanics of complex networks," Reviews of Modern Physics, vol. 74, no. 1, pp. 47-97, 2002.

[45] L.-X. Yang, M. Draief, and X. Yang, "The impact of the network topology on the viral prevalence: a node-based approach," PLoS ONE, vol. 10, no. 7, Article ID e0134507, 2015.

[46] L.-X. Yang, X. Yang, and Y. Wu, “The impact of patch forwarding on the prevalence of computer virus: a theoretical assessment approach," Applied Mathematical Modelling. Simulation and Computation for Engineering and Environmental Systems, vol. 43, pp. 110-125, 2017. 


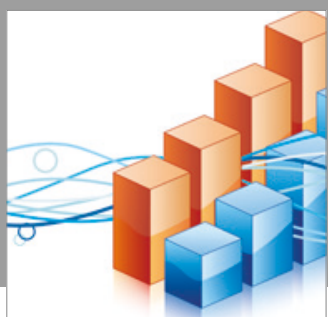

Advances in

Operations Research

vatersals

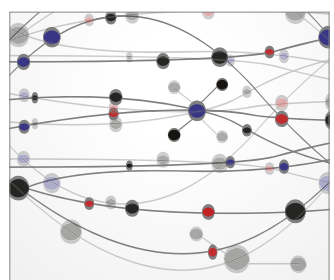

\section{The Scientific} World Journal
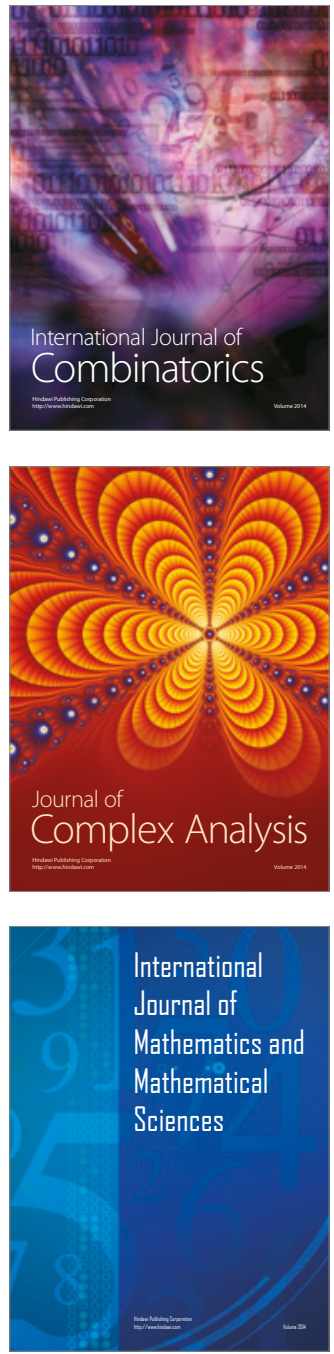
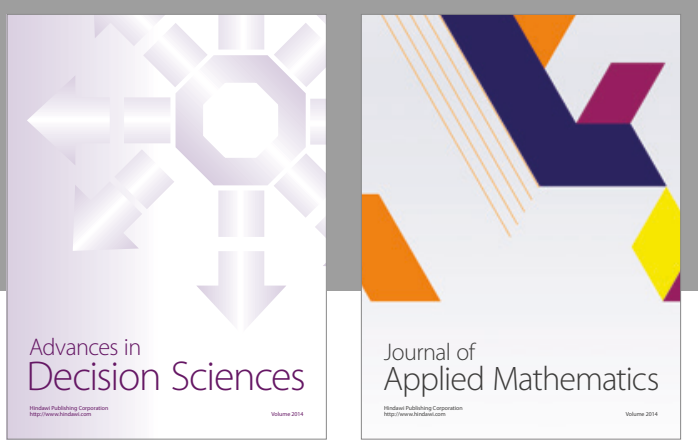

Algebra

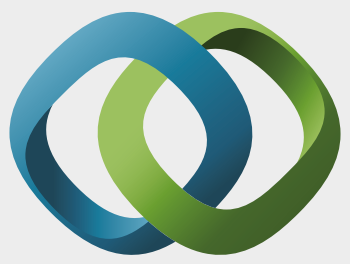

\section{Hindawi}

Submit your manuscripts at

https://www.hindawi.com
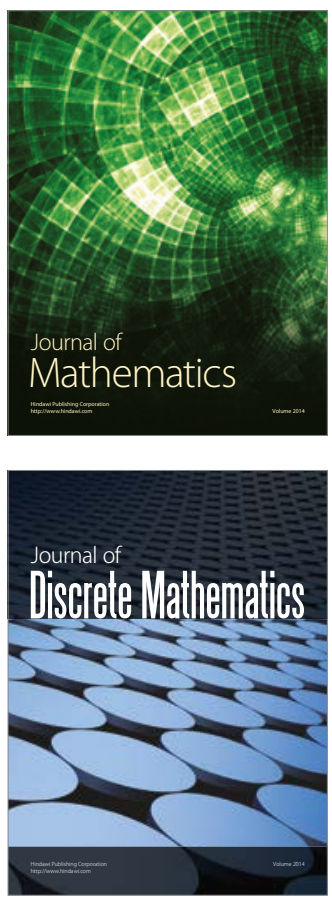

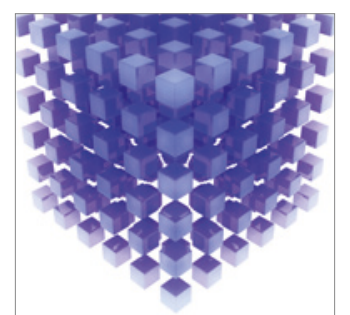

Mathematical Problems in Engineering
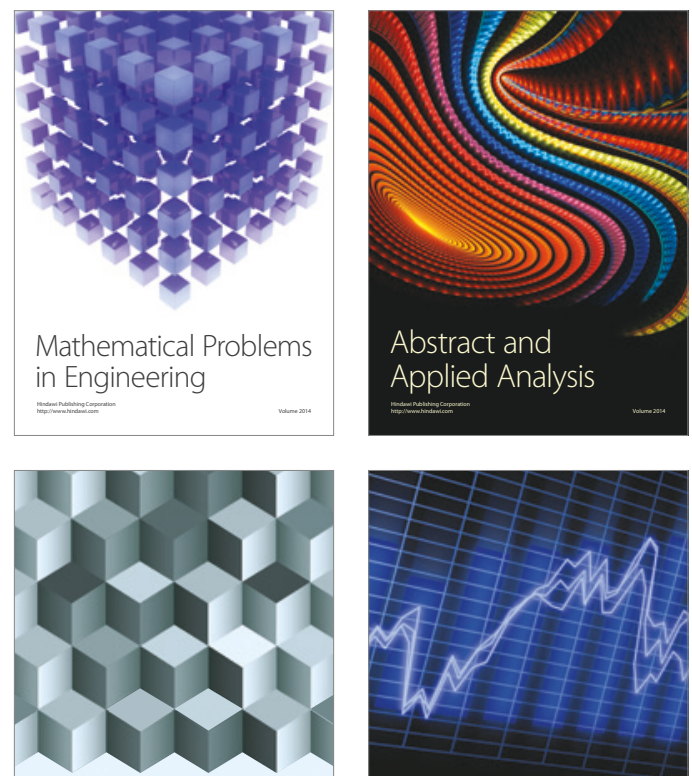

Journal of

Function Spaces

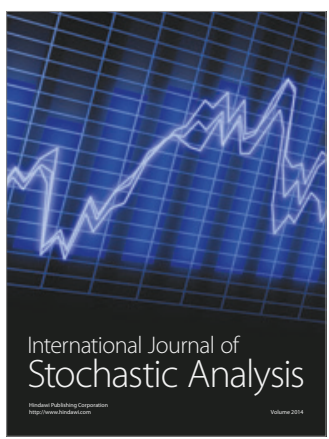

Probability and Statistics
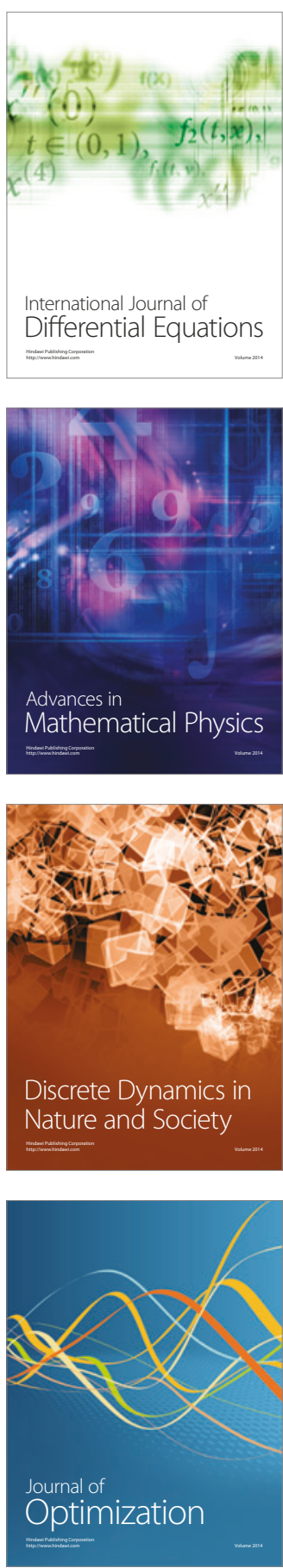